\title{
Understanding Proton Transfer in Non-aqueous Biopolymers based on Helical Peptides: A Quantum Mechanical Study
}

\author{
Jiang Bian ${ }^{1}$, Anthony $\mathrm{Cruz}^{1}$, Gabriel Lopez-Morales ${ }^{1}$, Anton Kyrylenko ${ }^{1}$, Donna \\ McGregor $^{1}$, and Gustavo Lopez ${ }^{2}$ \\ ${ }^{1}$ Lehman College \\ ${ }^{2}$ Lehman College - CUNY
}

January 5, 2022

\begin{abstract}
Histidine (an imidazole-based amino acid) is a promising building block for short aromatic peptides containing a proton donor/acceptor moiety. Previous studies have shown that polyalanine helical peptides substituted at regular intervals with histidine residues exhibit both structural stability as well as high proton affinity and high conductivity. Here, we present first-principle calculations of non-aqueous histidine-containing 310-, ?? and ?-helices and show that they are able to form hydrogen-bonded networks mimicking proton wires that have the ability to shuttle protons via the Grotthuss shuttling mechanism. The formation of these wires enhances the stability of the helices, and our structural characterizations confirm that the secondary structures are conserved despite distortions of the backbones. In all cases, the helices exhibit high proton affinity and proton transfer barriers on the order of $1^{\sim} 4 \mathrm{kcal} / \mathrm{mol}$. Zero-point energy calculations suggest that for these systems, ground state vibrational energy can provide enough energy to cross the proton transport energy barrier. Additionally, ab initio molecular dynamics results suggests that the protons are transported unidirectionally through the wire at a rate of approximately $2 \AA$ every 20 fs. These results demonstrate that efficient deprotonation-controlled proton wires can be formed using non-aqueous histidine-containing helical peptides.
\end{abstract}

\section{Hosted file}

PWP-IJQC.pdf available at https://authorea.com/users/454064/articles/551809-understandingproton-transfer-in-non-aqueous-biopolymers-based-on-helical-peptides-a-quantummechanical-study 